\title{
Optimal domains for the kernel operator associated with Sobolev's inequality
}

\author{
by \\ Guillermo P. Curbera (Sevilla) and Werner J. Ricker (Eichstätt)
}

\begin{abstract}
Refinements of the classical Sobolev inequality lead to optimal domain problems in a natural way. This is made precise in recent work of Edmunds, Kerman and Pick; the fundamental technique is to prove that the (generalized) Sobolev inequality is equivalent to the boundedness of an associated kernel operator on $[0,1]$. We make a detailed study of both the optimal domain, providing various characterizations of it, and of properties of the kernel operator when it is extended to act in its optimal domain. Several results are devoted to identifying the maximal rearrangement invariant space inside the optimal domain. The methods and techniques used involve interpolation theory, Banach function spaces and vector integration.
\end{abstract}

Introduction. In 1938 Sobolev proved the following inequality, valid for differentiable functions $f$ on a bounded domain $\Omega$ in $\mathbb{R}^{n}$ with $n \geq 2$ :

$$
\|f\|_{L^{q}(\Omega)} \leq C\|\mid \nabla f\|_{L^{p}(\Omega)}, \quad f \in C_{0}^{1}(\Omega),
$$

where $1<p<n, q:=n p /(n-p)$ and $C>0$ is a constant depending on $p$ and $n$. In the limiting case, when $p=n$, the result fails, i.e. we cannot take $q=\infty$. Indeed, in [25] Trudinger showed

$$
\|f\|_{L_{\varphi}(\Omega)} \leq C\|\mid \nabla f\|_{L^{n}(\Omega)}, \quad f \in C_{0}^{1}(\Omega),
$$

where $L_{\varphi}(\Omega)$ is the Orlicz space given by $\varphi(t)=\exp \left(t^{n^{\prime}}\right)-1$ and $n^{\prime}:=$ $n /(n-1)$ is the conjugate exponent of $n$. This result opened the way to refining Sobolev's inequality; one seeks a smaller range space for a given fixed domain space, or a bigger domain space for a given fixed range space. Thereby, the problems arise of determining the optimal range space and the optimal domain space for Sobolev's inequality. In this regard, see also the recent work [20].

2000 Mathematics Subject Classification: Primary 47B38, 46E30; Secondary 47G10, $28 \mathrm{~B} 05$.

Key words and phrases: Sobolev imbeddings, optimal domains, vector measures.

The authors gratefully acknowledge the support of the Katholische Universität Eichstätt-Ingolstadt (Germany) and D.G.I. \#BFM2000-0206-C04-02 (Spain). 
Such optimality problems have to be considered within a particular class of spaces. We may choose the class of Lebesgue spaces. In this context, for inequality (1) the space $L^{q}(\Omega)$ with $q:=n p /(n-p)$ is the optimal range space given the fixed domain space $L^{p}(\Omega), 1<p<n$. In the class of Orlicz spaces, the space $L_{\varphi}(\Omega)$ with $\varphi(t)=\exp \left(t^{n^{\prime}}\right)-1$ and $n^{\prime}:=n /(n-1)$ is the optimal range space for the fixed domain space $L^{n}(\Omega)$ (see [11]). The optimal range problem within the class of Orlicz spaces has been studied by Cianchi [4].

In the other direction, Edmunds, Kerman and Pick studied the optimal domain problem, for inequality (1), within the class of rearrangement invariant (briefly, r.i.) spaces [10]. They consider r.i. norms $\varrho_{\mathrm{R}}$ and $\varrho_{\mathrm{D}}$ and a generalized Sobolev inequality

$$
\varrho_{\mathrm{R}}\left(f^{*}\right) \leq C \varrho_{\mathrm{D}}\left(|\nabla f|^{*}\right), \quad f \in C_{0}^{1}(\Omega),
$$

where $f^{*}$ and $|\nabla f|^{*}$ are, respectively, the decreasing rearrangements of $f$ and its gradient $|\nabla f|$. Using results of Talenti, they show (for $|\Omega|=1$ ) that (2) is equivalent to boundedness of the kernel operator $T$ associated with Sobolev's inequality, namely

$$
T f(t)=\int_{t}^{1} f(s) s^{(1 / n)-1} d s, \quad t \in[0,1],
$$

acting between the function spaces on $[0,1]$ with norms $\varrho_{\mathrm{R}}$ and $\varrho_{\mathrm{D}}$, that is, $\varrho_{\mathrm{R}}(T f) \leq K \varrho_{\mathrm{D}}(f)\left[10\right.$, Theorem 6.1]. For a fixed r.i. norm $\varrho_{\mathrm{R}}$ they determine the smallest norm $\varrho_{\mathrm{D}}$ for which (2) holds (i.e. the optimal domain) [10, Theorem 4.1], namely

$$
\varrho_{\mathrm{D}}(f):=\varrho_{\mathrm{R}}\left(t \mapsto \int_{t}^{1}|f(s)| s^{(1 / n)-1} d s\right) .
$$

Our aim is to identify such optimal domains, and related spaces, and to study their properties in terms of the properties of the range space $X$. Given a r.i. space $X$ on $[0,1]$ with norm $\varrho$, i.e., $X=\left(L^{1}, L^{\infty}\right)_{\varrho}$, let $[T, X]$ denote the optimal lattice domain corresponding to the imbedding (2), that is, the maximal Banach function space on $[0,1]$ to which $T$ can be extended as a continuous linear operator, with values still in $X$ [8, Section 3]. Various equivalent descriptions of the optimal domain $[T, X]$ can be formulated. The kernel

$$
K(t, s):=s^{(1 / n)-1} \chi_{[t, 1]}(s), \quad(t, s) \in[0,1] \times(0,1],
$$

generates the operator (3). Since $K$ is non-negative, $[T, X]=\{f: T|f| \in X\}$ [8, Proposition 5.2]. Moreover, $K$ satisfies the conditions of [8, Theorem 5.12] and so

$$
[T, X]=\left(L^{1}\left(s^{1 / n} d s\right), L^{1}\left(s^{(1 / n)-1} d s\right)\right)_{\varrho},
$$

where this last interpolation space is obtained via the $\mathrm{K}$-functional and the r.i. norm $\varrho[2, \mathrm{~V} .1]$. 
An alternative description of the optimal domain space $[T, X]$ is possible in terms of the $L^{1}$-space of the associated vector measure $\nu_{X}$. Indeed, put $f=\chi_{A}$ in (3), for $A \in \mathcal{B}$ (the $\sigma$-algebra of all Borel sets in $[0,1]$ ). Then $T\left(\chi_{A}\right) \in C([0,1]) \subset X$ and the $X$-valued set function

$$
\nu_{X}: A \mapsto \nu_{X}(A):=T\left(\chi_{A}\right)
$$

is countably additive, i.e. it is a vector measure in $X$. Let $L^{1}\left(\nu_{X}\right)$ denote the space of all $\nu_{X}$-integrable functions, equipped with the topology of convergence in mean (see Section 1). For general $X$-valued measures $\nu$ associated with a kernel operator $S$, we always have $L^{1}(\nu) \subset[S, X]$, but the inclusion may be proper [8, Remark 5.3]. Equality occurs whenever $X$ has absolutely continuous (briefly, a.c.) norm [8, Proposition 5.2]. It will be shown (Proposition 3.5), for the particular kernel operator (3) associated with Sobolev's inequality, that

$$
[T, X]=L^{1}\left(\nu_{X}\right)
$$

without any restrictions on $X$. With this result, we will be able to apply the theory and techniques used in our study of $L^{1}\left(\nu_{X}\right)$ (see [8]) to the space $[T, X]$. The important point in this regard is that the integration operator $f \mapsto \int f d \nu_{X}$, for $f \in L^{1}\left(\nu_{X}\right)$, is precisely the restriction of the kernel operator $T$ given in (3) to $L^{1}\left(\nu_{X}\right)$.

This provides a strong motivation to better understand the Banach function spaces $L^{1}\left(\nu_{X}\right)$, the associated integration operator, and the connections to properties of the underlying r.i. space $X$. The optimal domain space $[T, X]=L^{1}\left(\nu_{X}\right)$ need not be r.i. The final sections contain various results identifying the largest r.i. space inside $[T, X]$. For instance, it is shown that if the dilation exponents of the function $\varphi$ determining the Marcinkiewicz space $X:=M_{\varphi}$ satisfy $1 / n<\gamma_{\varphi} \leq \delta_{\varphi}<1$, then the largest r.i. space inside $[T, X]$ is the Marcinkiewicz space $M_{\Phi}$, where $\Phi$ is equivalent to $t \mapsto t^{-1 / n} \varphi(t)$; the space $[T, X]$ itself is not r.i., that is, the inclusion $M_{\Phi} \subset[T, X]$ is proper.

1. Preliminaries. A r.i. space $X$ on $[0,1]$ is a Banach space of integrable functions on $[0,1]$ which contains the simple functions, has the property that $g \in X$ and $\|g\| \leq\|f\|$ whenever $f \in X$ and $|g| \leq|f|$, satisfies the Fatou property, and has the property that whenever $f \in X$ and $g$ is equimeasurable with $f$, then $g \in X$ and $\|g\|=\|f\|$. In particular, if $f^{*}$ is the decreasing rearrangement of $f \in X$, then $f^{*} \in X$ with $\left\|f^{*}\right\|=\|f\|[2$, I.1.1; II.4.1]. Hence, $L^{\infty}([0,1]) \subset X \subset L^{1}([0,1])$ continuously. The closure of $L^{\infty}([0,1])$ in $X$ is denoted by $X_{\mathrm{b}}$; it is again a r.i. space. The associate space $X^{\prime}$ of $X$ is the space of functions $g$ on $[0,1]$ such that $f g \in L^{1}([0,1])$ for every $f \in X[2$, II.4.2]. 
Let $\varphi$ be an increasing, concave function on $[0,1]$ with $\varphi(0)=0$. The Lorentz space $\Lambda_{\varphi}$ associated to $\varphi$, for short $\Lambda$, is defined by

$$
\Lambda_{\varphi}:=\left\{f:\|f\|_{\Lambda_{\varphi}}:=\int_{0}^{1} f^{*}(s) d \varphi(s)<\infty\right\},
$$

and the Marcinkiewicz space $M_{\varphi}$ associated to $\varphi$ is given by

$$
M_{\varphi}:=\left\{f:\|f\|_{M_{\varphi}}:=\sup _{0<t \leq 1} \frac{1}{\varphi(t)} \int_{0}^{t} f^{*}(s) d s<\infty\right\} .
$$

Both are r.i. spaces and $\left(\Lambda_{\varphi}\right)^{\prime}=M_{\varphi}[13$, p. 112]. We also consider such spaces for a quasiconcave function $\varphi$ (concavity is replaced with $\varphi(t) / t$ decreasing) since, in this case, there is an equivalent concave function [13, p. 49]. If two functions are equivalent [13, p. 48], then the corresponding Lorentz and Marcinkiewicz spaces are isomorphic.

The fundamental function $\varphi_{X}$ of a r.i. space $X$ is defined by $\varphi_{X}(s):=$ $\left\|\chi_{[0, s]}\right\|_{X}$. It is increasing, quasiconcave with $\varphi_{X}\left(0^{+}\right):=\lim _{t \rightarrow 0^{+}} \varphi_{X}(t) \geq 0$. We may assume $\varphi_{X}$ is concave [2, II.5.11]. The space $\Lambda_{X}:=\Lambda_{\varphi}$ (for $\varphi=$ $\left.\varphi_{X}\right)$ is the smallest r.i. space having fundamental function $\varphi_{X}$. The space $M_{X}:=M_{\varphi}\left(\right.$ for $\left.\varphi(t)=t / \varphi_{X}(t)\right)$ is the largest r.i. space having fundamental function $\varphi_{X}$. By a result of Semenov, $\Lambda_{X} \hookrightarrow X \hookrightarrow M_{X}$ [13, pp. 118-119]. The space $X$ is isomorphic to $L^{\infty}([0,1])$ if and only if $\varphi_{X}\left(0^{+}\right)>0[23$, Lemma 3, p. 220].

We briefly recall the theory of integration of real functions with respect to a vector measure, initially due to Bartle, Dunford and Schwartz [1]. Let $(\Omega, \Sigma)$ be a measurable space, $X$ a Banach space and $\nu: \Sigma \rightarrow X$ a countably additive vector measure. Let $X^{*}$ be the dual space of $X$ and, for each $x^{*} \in$ $X^{*}$, denote the $\mathbb{R}$-valued measure $A \mapsto\left\langle x^{*}, \nu(A)\right\rangle$ by $x^{*} \nu$ and its variation measure by $\left|x^{*} \nu\right|$. A measurable function $f: \Omega \rightarrow \mathbb{R}$ is integrable with respect to $\nu$ if $f \in L^{1}\left(\left|x^{*} \nu\right|\right)$ for every $x^{*} \in X^{*}$, and for each $A \in \Sigma$ there exists a vector in $X$ (denoted by $\int_{A} f d \nu$ ) such that $\left\langle\int_{A} f d \nu, x^{*}\right\rangle=\int_{A} f d x^{*} \nu$ for every $x^{*} \in X^{*}$ (see [14]). The $\nu$-integrable functions form a linear space in which

$$
\|f\|_{\nu}:=\sup \left\{\int|f| d\left|x^{*} \nu\right|: x^{*} \in X^{*},\left\|x^{*}\right\| \leq 1\right\}
$$

is a seminorm. A set $A \in \Sigma$ is $\nu$-null if $\left|x^{*} \nu\right|(A)=0$ for every $x^{*} \in X^{*}$.

Identifying functions which differ on a $\nu$-null set, we obtain a Banach space (of classes) of $\nu$-integrable functions, denoted by $L^{1}(\nu)$. It is a Banach function space [5, Theorem 1] for the $\nu$-a.e. order, and has a.c. norm, i.e., order bounded, increasing sequences are norm convergent. Simple functions are dense in $L^{1}(\nu)$ and the $\nu$-essentially bounded functions are contained in $L^{1}(\nu)$. The integration operator $I_{\nu}$ from $L^{1}(\nu)$ to $X$ is defined by $f \mapsto \int f d \nu$. It is continuous, linear and of norm at most one. No assumptions have been 
made on the variation measure $|\nu|$ of $\nu$ in the definition of $L^{1}(\nu)$. In general $L^{1}(|\nu|) \subset L^{1}(\nu)$ [15, Theorem 4.1]. For further details concerning $L^{1}(\nu)$ see [5], [6], [18]. Such spaces $L^{1}(\nu)$ can be quite different from the classical $L^{1}$-spaces of scalar measures and may be difficult to identify explicitly [7]. Indeed, every Banach lattice with a.c. norm and having a weak unit (e.g. $\left.L^{2}([0,1])\right)$ is the $L^{1}$-space of some vector measure [5, Theorem 8$]$.

In general we will follow the notation in [2], [9], [13] and [16].

2. Basic results for $X=L^{\infty}([0,1])$. To undertake a more detailed study of the kernel operator $T$ associated to Sobolev's inequality, we first consider the case $X=L^{\infty}([0,1])$.

The vector function $F$ associated to the kernel (4) is given by

$$
F(s)=s^{(1 / n)-1} \chi_{[0, s]}(t), \quad s \in(0,1], t \in[0,1] .
$$

It is $L^{\infty}([0,1])$-valued and satisfies $\|F(s)\|_{\infty}=s^{(1 / n)-1}$. The vector measure $\nu$ (see $(5))$ associated to $T$ for $\left.X=L^{\infty}([0,1])\right)$ is given by

$$
\nu(A)(t)=\int_{t}^{1} \chi_{A}(s) s^{(1 / n)-1} d s, \quad 0 \leq t \leq 1, A \in \mathcal{B} .
$$

It takes its values in $C([0,1]) \subset L^{\infty}([0,1])$ and is $\sigma$-additive because

$$
\|\nu(A)\|_{\infty}=\left\|\int_{(\cdot)}^{1} \chi_{A}(s) s^{(1 / n)-1} d s\right\|_{\infty}=\int_{A} s^{(1 / n)-1} d s .
$$

The next result collects some basic properties of $\nu$. For the notion of a Banach space valued function on $[0,1]$ being Bochner or Pettis $\lambda$-integrable (for Lebesgue measure $\lambda$ ) we refer to [9].

Proposition 2.1. Let $\nu$ be the $L^{\infty}([0,1])$-valued vector measure $(7)$.

(a) The measure $\nu$ has bounded variation, with $|\nu|(A)=\int_{A} s^{(1 / n)-1} d s$ for $A \in \mathcal{B}$, and the range of $\nu$ is relatively compact in $L^{\infty}([0,1])$.

(b) The function $F:[0,1] \rightarrow L^{\infty}([0,1])$ is Pettis $\lambda$-integrable and

$$
\nu(A)=\int_{A} F d \lambda, \quad A \in \mathcal{B} .
$$

(c) The function $F$ has non-separable range, and hence is not strongly measurable. In particular, $F$ is not Bochner $\lambda$-integrable.

(d) $L^{1}(\nu)$ is equal to $L^{1}(|\nu|)=L^{1}\left(s^{(1 / n)-1} d s\right)$. Moreover, the optimal domain is given by $\left[T, L^{\infty}([0,1])\right]=L^{1}(\nu)$.

Proof. (a) The first claim is immediate from (8). As for the second claim, it is clear from $(7)$ that $\nu$ takes its values in $C([0,1])$ and satisfies $\nu(A)(t)=$ $\int_{A} K(t, s) d s$ for $t \in[0,1]$ and $A \in \mathcal{B}$, where $K$ is the kernel (4). The result then follows from [8, Proposition 4.1]. 
(b) We first verify that $F$ is weakly integrable. Let $x^{*} \in L^{\infty}([0,1])^{*}$; we can suppose that $x^{*} \geq 0$. The function $s \in(0,1] \mapsto\left\langle F(s), x^{*}\right\rangle=$ $s^{(1 / n)-1} x^{*}\left(\chi_{[0, s]}\right)$ is measurable. Its $\lambda$-integrability follows from

$$
\left|\left\langle F(s), x^{*}\right\rangle\right| \leq\|F(s)\|_{\infty}\left\|x^{*}\right\|=s^{(1 / n)-1}\left\|x^{*}\right\| .
$$

Hence, $F:(0,1] \rightarrow L^{\infty}([0,1])$ is Dunford-integrable with respect to $\lambda[9$, pp. 52-56]. This implies that the operator $S: L^{\infty}([0,1])^{*} \rightarrow L^{1}([0,1])$ given by $S x^{*}=\left\langle F(\cdot), x^{*}\right\rangle$ is well defined. Moreover, (9) shows that $S$ is weakly compact, since the image of the unit ball is uniformly integrable. Hence, since $F$ is defined on a separable measure space, it follows by [12, Corollary 4] that $F$ is Pettis $\lambda$-integrable.

To verify $\nu=\int F d \lambda$, define $m(A)=\int_{A} F d \lambda$ for $A \in \mathcal{B}$. As $F$ is Pettis $\lambda$-integrable, the Orlicz-Pettis theorem ensures $m$ is $\sigma$-additive. Fix $A \in \mathcal{B}$ and let $\psi \in L^{1}([0,1])$. By Fubini's theorem $\langle\nu(A), \psi\rangle=\langle m(A), \psi\rangle$. But $L^{1}([0,1])$ separates points of $L^{\infty}([0,1])$ and so $\nu=m$.

(c) The function $F$ is not strongly measurable, because its range is not separable. Indeed, let $0<s_{1}<s_{2}$; then

$$
\begin{aligned}
\left\|F\left(s_{1}\right)-F\left(s_{2}\right)\right\|_{\infty} & =\left\|s_{1}^{(1 / n)-1} \chi_{\left(0, s_{1}\right]}-s_{2}^{(1 / n)-1} \chi_{\left(0, s_{2}\right]}\right\|_{\infty} \\
& \geq s_{2}^{(1 / n)-1}\left\|\chi_{\left[s_{1}, s_{2}\right]}\right\|_{\infty} \geq 1 .
\end{aligned}
$$

(d) By (a) we have $L^{1}(|\nu|)=L^{1}\left(s^{(1 / n)-1} d s\right)$. Recall that $L^{1}(|\nu|) \subset$ $L^{1}(\nu)$. Let $f \in L^{1}(\nu)$. Note that $\nu$ is non-negative, i.e. $\nu(A)(t) \geq 0$ for all $t$. Then $\int|f| d \nu \geq 0$, and so

$$
\begin{aligned}
\int_{0}^{1}|f(s)| s^{(1 / n)-1} d s & =\left\|\int_{(\cdot)}^{1}|f(s)| s^{(1 / n)-1} d s\right\|_{\infty}=\left\|\int|f| d \nu\right\|_{\infty} \\
& =\sup _{0 \leq x^{*},\left\|x^{*}\right\| \leq 1}\left\langle\int|f| d \nu, x^{*}\right\rangle \\
& =\sup _{0 \leq x^{*},\left\|x^{*}\right\| \leq 1} \int|f| d x^{*} \nu=\|f\|_{\nu}<\infty .
\end{aligned}
$$

As noted earlier, $L^{1}(\nu) \subset\left[T, L^{\infty}([0,1])\right]$ always holds. Let $f \in$ $\left[T, L^{\infty}([0,1])\right]$. Then $T|f| \in L^{\infty}([0,1])$ and so, from (3), we have $0 \leq$ $\int_{t}^{1}|f(s)| s^{(1 / n)-1} d s \leq\|T|f|\|_{\infty}$ for all $0<t \leq 1$. Hence, $f \in L^{1}\left(s^{(1 / n)-1} d s\right)$, which shows that $f \in L^{1}(\nu)$.

Recall that the integration map $I_{\nu}$ is given by

$$
t \mapsto \int_{t}^{1} f(s) s^{(1 / n)-1} d s, \quad t \in[0,1],
$$

for every $f \in L^{1}(\nu)=L^{1}\left(s^{(1 / n)-1} d s\right)$. By $(10), I_{\nu}$ is a positive operator from $L^{1}(\nu)$ into $L^{\infty}([0,1])$. Of course, $I_{\nu}$ is the kernel operator $T$ restricted 
to $L^{1}(\nu)$. The space of absolutely continuous functions on $[0,1]$ which vanish at 1 is denoted by $\mathrm{AC}_{1}([0,1])$.

Proposition 2.2. Let $I_{\nu}: L^{1}(\nu) \rightarrow L^{\infty}([0,1])$ be the map $(10)$.

(a) $I_{\nu}$ is injective.

(b) The range $I_{\nu}\left(L^{1}(\nu)\right)=\mathrm{AC}_{1}([0,1])$.

(c) $I_{\nu}$ is not weakly compact, and hence also not compact.

Proof. (a) Let $f \in L^{1}(\nu)$. Then $g(s):=s^{(1 / n)-1} f(s)$ belongs to $L^{1}([0,1])$ by Proposition 2.1(d). Suppose $I_{\nu}(f)=0$ in $L^{\infty}([0,1])$. It is clear from $(10)$ that $I_{\nu}(f) \in \mathrm{AC}_{1}([0,1])$ and so $I_{\nu}(f)(t)=0$ for every $t \in[0,1]$. For every $0 \leq a<b \leq 1$, we have $\int_{0}^{1} \chi_{[a, b]} g d \lambda=I_{\nu}(f)(b)-I_{\nu}(f)(a)=0$. Hence, $g=0$ a.e. in $[0,1]$. We conclude $f$ is zero a.e. on $[0,1]$.

(b) It was already noted in (a) that $I_{\nu}\left(L^{1}(\nu)\right) \subset \mathrm{AC}_{1}([0,1])$. So, let $h \in \mathrm{AC}_{1}([0,1])$. Define $f(s):=-s^{1-(1 / n)} h^{\prime}(s)$ for a.e. $s \in[0,1]$. Since

$$
\int_{0}^{1}|f(s)| s^{(1 / n)-1} d s=\int_{0}^{1}\left|h^{\prime}(s)\right| d s<\infty,
$$

it follows from Proposition 2.1(d) that $f \in L^{1}(\nu)$. According to (10) we have $I_{\nu}(f)(t)=\int_{t}^{1}-h^{\prime}(s) d s=h(t)$ for every $t \in[0,1]$. This establishes that $I_{\nu}$ maps $L^{1}(\nu)$ onto $\mathrm{AC}_{1}([0,1])$.

(c) Suppose $I_{\nu}$ is weakly compact. By Proposition 2.1(d) the functions $f_{k}(s):=k s^{1-(1 / n)} \chi_{[0,1 / k]}(s)$, for $s \in[0,1]$ and $k \geq 1$, all have norm one in $L^{1}(\nu)$. Since $I_{\nu}$ takes its values in the closed subspace $C([0,1])$ of $L^{\infty}([0,1])$, it follows that $\left\{I_{\nu}\left(f_{k}\right)\right\}$ is relatively weakly compact in $C([0,1])$. By passing to a subsequence, if needed, we may assume (by the Eberlein-Shmul'yan theorem) that $\left\{I_{\nu}\left(f_{k}\right)\right\}$ converges weakly in $C([0,1])$. Considering the Dirac point measures and using (10), which implies $I_{\nu}\left(f_{k}\right)(t)=0$ for all $t \in[1 / k, 1]$ and $I_{\nu}\left(f_{k}\right)(0)=1$ (for all $k \geq 1$ ), it follows that the weak limit of $\left\{I_{\nu}\left(f_{k}\right)\right\}$ must be the function $\chi_{\{1\}}$, which is not in $C([0,1])$. So, $I_{\nu}$ is not weakly compact.

REMARK 2.3. (a) Propositions 2.1(a) and 2.2(c) should be compared.

(b) Since the restriction of $I_{\nu}$ to $L^{\infty}([0,1])$ coincides with $T$, it follows from Proposition 2.2(b) that $T$ maps $L^{\infty}([0,1])$ into $C([0,1])$. Ascoli's theorem implies that $T: L^{\infty}([0,1]) \rightarrow L^{\infty}([0,1])$ is compact.

3. Basic results for $X \not L^{\infty}([0,1])$. Let $X$ be a r.i. space on $[0,1]$. Since $L^{\infty}([0,1])$ imbeds continuously into $X$, the function $F$ in $(6)$ takes its values in $X$ and the measure $\nu$ in (7) is $\sigma$-additive as an $X$-valued measure. We will denote $F$ by $F_{X}$ and $\nu$ by $\nu_{X}$ to indicate the space where the values are taken. 
Proposition 3.1. Let $X$ be a r.i. space not isomorphic to $L^{\infty}([0,1])$.

(a) The function $F_{X}:[0,1] \rightarrow X$ is Bochner $\lambda$-integrable and $\nu_{X}(A)=$ $\int_{A} F_{X} d \lambda$ for $A \in \mathcal{B}$. Consequently, $\nu_{X}$ has bounded variation, given by

$$
\left|\nu_{X}\right|(A)=\int_{A} s^{(1 / n)-1} \varphi_{X}(s) d s, \quad A \in \mathcal{B} .
$$

(b) The range $\nu_{X}(\mathcal{B}) \subset X_{\mathrm{b}}$ and is a relatively compact subset of $X$.

Proof. (a) To see that $F_{X}$ is strongly measurable, we check that it is continuous on $(0,1]$. Let $0<s_{1}<s_{2}$. Then

$$
\begin{aligned}
\left\|F_{X}\left(s_{1}\right)-F_{X}\left(s_{2}\right)\right\|_{X} & =\left\|s_{1}^{(1 / n)-1} \chi_{\left(0, s_{1}\right]}-s_{2}^{(1 / n)-1} \chi_{\left(0, s_{2}\right]}\right\|_{X} \\
& =\left\|\left(s_{1}^{(1 / n)-1}-s_{2}^{(1 / n)-1}\right) \chi_{\left(0, s_{1}\right]}-s_{2}^{(1 / n)-1} \chi_{\left(s_{1}, s_{2}\right]}\right\|_{X} \\
& \leq\left(s_{1}^{(1 / n)-1}-s_{2}^{(1 / n)-1}\right) \varphi_{X}(1)+s_{2}^{(1 / n)-1} \varphi_{X}\left(s_{2}-s_{1}\right),
\end{aligned}
$$

and this last term tends to 0 as $s_{2}-s_{1} \rightarrow 0$, since $\varphi_{X}\left(0^{+}\right)=0$. Now

$$
\int_{(0,1]}\left\|F_{X}(s)\right\|_{X} d s=\int_{(0,1]} s^{(1 / n)-1} \varphi_{X}(s) d s,
$$

which is finite as $\varphi_{X}$ is bounded. So, $F_{X}$ is Bochner $\lambda$-integrable.

To verify $\nu_{X}=\int F_{X} d \lambda$, define $m(A)=\int_{A} F_{X} d \lambda$ for $A \in \mathcal{B}$. Fix $A \in \mathcal{B}$ and let $\psi \in X^{\prime}$. As in the proof of Proposition 2.1(b) we have $\left\langle\nu_{X}(A), \psi\right\rangle=$ $\langle m(A), \psi\rangle$. But $X^{\prime}$ separates points of $X$ and so $\nu_{X}=m$.

(b) Note that $\nu_{X}(\mathcal{B}) \subset C([0,1]) \subset X_{\mathrm{b}}$. Relative compactness of $\nu_{X}(\mathcal{B})$ follows from Proposition 2.1(a).

REMARK 3.2. The proof of Proposition 3.1(a) implies, for $X=L^{1}([0,1])$ and with the definition $F_{L^{1}}(0):=0$, that $F_{L^{1}}:[0,1] \rightarrow L^{1}([0,1])$ is continuous. So, its range is a compact subset of $L^{1}([0,1])$. It then follows from Proposition 3.1(a) and [9, Theorem 2, p. 68] that the kernel operator $T: L^{1}([0,1]) \rightarrow L^{1}([0,1])$ is compact.

One aim is to describe and identify properties of the optimal domain $[T, X]$. Because of Proposition 3.5 below, this means a detailed study of $L^{1}\left(\nu_{X}\right)$. We first collect some basic properties of these spaces.

Proposition 3.3. Let $X$ be a r.i. space.

(a) $L^{1}\left(\nu_{X}\right)$ is a separable Banach function space with a.c. norm.

(b) $L^{1}\left(\nu_{X}\right)=L^{1}\left(\nu_{X_{\mathrm{b}}}\right)$.

(c) $L^{1}\left(\nu_{X}\right)$ is weakly sequentially complete.

Proof. (a) That $L^{1}\left(\nu_{X}\right)$ has a.c. norm is a general fact from vector integration theory [5, Theorem 1]. Since $\mathcal{B}$ is countably generated the separability follows [21, Proposition 2]. 
(b) Since $\nu_{X}(\mathcal{B}) \subset X_{\mathrm{b}}$ (Proposition 3.1(b)), it is known that in this case $L^{1}\left(\nu_{X}\right)=L^{1}\left(\nu_{X_{\mathrm{b}}}\right)[22$, Lemma 2.3$]$.

(c) For $X=L^{\infty}([0,1])$, the statement is clear from Proposition 2.1(d). So, we assume that $X \not L^{\infty}([0,1])$. If $Y$ is a Banach function space, then $Y$ is sequentially complete for the topology $\sigma\left(Y, Y^{\prime}\right)$, where $Y^{\prime}$ is the associate space of $Y$ [2, I.5.3]. Since $X$ is a r.i. space for which $\varphi_{X}\left(0^{+}\right)=0$, we have $X_{b}^{\prime}=X^{\prime}=X_{\mathrm{b}}^{*}\left[2\right.$, II.5.5]. Hence, $X_{\mathrm{b}}$ is weakly sequentially complete, and thus so is $L^{1}\left(\nu_{X_{\mathrm{b}}}\right)$ [5, Theorem 3], and then also $L^{1}\left(\nu_{X}\right)$ by part (b).

Given a r.i. space $X$, it is useful to be able to decide which measurable functions $f$ on $[0,1]$ belong to the optimal domain space $[T, X]$. Since $[T, X]=L^{1}\left(\nu_{X}\right)$ (see Proposition 3.5 below) the following result provides useful criteria for deciding membership of $[T, X]$.

Proposition 3.4. Let $X$ be a r.i. space on $[0,1]$ not isomorphic to $L^{\infty}([0,1])$. Let $f:[0,1] \rightarrow \mathbb{R}$ be a measurable function.

(a) The following statements are equivalent:

(i) $f \in L^{1}\left(\nu_{X}\right)$.

(ii) The function $f F_{X}:[0,1] \rightarrow X$ is Pettis $\lambda$-integrable.

(iii) $\int_{0}^{1}|f| d\left|x^{\prime} \nu_{X}\right|<\infty$ for every $x^{\prime} \in X^{\prime}$.

(iv) For every $g \in X^{\prime}$ which is non-negative and decreasing

$$
\int_{0}^{1}|f(s)| s^{(1 / n)-1} \int_{0}^{s} g(t) d t d s<\infty .
$$

(b) $f \in L^{1}\left(\left|\nu_{X}\right|\right)$ iff $f F_{X}:[0,1] \rightarrow X$ is Bochner $\lambda$-integrable.

Proof. (a) Given $x^{*} \in X^{*}$, it follows from Proposition 3.1(a) that

$$
\left|x^{*} \nu_{X}\right|(A)=\int_{A}\left|\left\langle F_{X}, x^{*}\right\rangle\right| d \lambda, \quad A \in \mathcal{B} .
$$

According to the definition [9, II.3], the function $f F_{X}$ is Pettis $\lambda$-integrable if $\left\langle f F_{X}, x^{*}\right\rangle \in L^{1}([0,1])$ for each $x^{*} \in X^{*}$ and for each $A \in \mathcal{B}$ there exists a vector in $X$ (denoted by $\int_{A} f F_{X} d \lambda$ ) such that $\left\langle\int_{A} f F_{X} d \lambda, x^{*}\right\rangle=$ $\int_{A} f\left\langle F_{X}, x^{*}\right\rangle d \lambda$ for $x^{*} \in X^{*}$. In view of (13) and the definition of $\nu_{X^{-}}$ integrability we see that (i) $\Leftrightarrow$ (ii).

Since $X^{\prime} \subset X^{*}$, it is clear that (i) $\Rightarrow$ (iii). So, suppose that (iii) holds. Since $\nu_{X}(\mathcal{B}) \subset X_{\mathrm{b}}$ and $X_{\mathrm{b}}^{*}=X^{\prime}[2$, II.5.5], we conclude that

$$
\int_{0}^{1}|f| d\left|u^{*} \nu_{X_{\mathrm{b}}}\right|<\infty, \quad u^{*} \in X_{\mathrm{b}}^{*} .
$$

But we have seen in the proof of Proposition 3.3(c) that $X_{\mathrm{b}}$ is weakly sequentially complete and hence cannot contain a copy of the sequence space $c_{0}$. 
A result of Lewis [15, Theorem 5.1], shows that (14) implies $f \in L^{1}\left(\nu_{X_{\mathrm{b}}}\right)$. Hence, by Proposition 3.3(b), also $f \in L^{1}\left(\nu_{X}\right)$.

Recall elements of $X^{\prime}$ are functions. Let $g \in X^{\prime}$. Since $X^{\prime}$ is r.i. also $g^{*} \in X^{\prime}$. By (13) and (6),

$$
\begin{aligned}
\left|g \nu_{X}\right|(A) & =\int_{A} s^{(1 / n)-1}\left|\left\langle\chi_{[0, s]}, g\right\rangle\right| d s=\int_{A} s^{(1 / n)-1}\left|\int_{0}^{s} g(t) d t\right| d s \\
& \leq \int_{A} s^{(1 / n)-1} \int_{0}^{s} g^{*}(t) d t d s=\left|g^{*} \nu_{X}\right|(A) .
\end{aligned}
$$

Hence, (iv) implies (iii). Since Proposition 3.1(a) implies

$$
\left\langle\nu_{X}, x^{\prime}\right\rangle(A)=\int_{A} s^{(1 / n)-1}\left\langle\chi_{[0, s]}, x^{\prime}\right\rangle d s \quad \text { for } A \in \mathcal{B},
$$

it is clear (iii) implies (iv).

(b) As $f$ is $\mathbb{R}$-valued and measurable and $F_{X}$ is strongly measurable, also $f F_{X}:[0,1] \rightarrow X$ is strongly measurable. So, $f F_{X}$ is Bochner $\lambda$-integrable iff

$$
\int_{0}^{1}\left\|f F_{X}\right\| d \lambda=\int_{0}^{1}|f(s)| \varphi_{X}(s) s^{(1 / n)-1} d s<\infty .
$$

In view of (11) this is the required equivalence.

As a consequence of the previous result we have the following important fact.

Proposition 3.5. Let $X$ be a r.i. space. Then $[T, X]=L^{1}\left(\nu_{X}\right)$. In particular, $[T, X]=\left[T, X_{\mathrm{b}}\right]$.

Proof. For $X=L^{\infty}([0,1])$, see Proposition 2.1(d).

When $X \not L^{\infty}([0,1])$, we only have to establish $[T, X] \subset L^{1}\left(\nu_{X}\right)$. So, let $f \in[T, X]$. Then $T|f| \in X$. Let $g \in X^{\prime}$ be non-negative. Then, by (3),

$$
\begin{aligned}
\int_{0}^{1}|f(s)| s^{(1 / n)-1} \int_{0}^{s} g(t) d t d s & =\int_{0}^{1} g(t) \int_{t}^{1}|f(s)| s^{(1 / n)-1} d s d t \\
& =\langle g, T|f|\rangle<\infty,
\end{aligned}
$$

since $g \in X^{\prime}$ and $T|f| \in X$. Condition (12) implies $f \in L^{1}\left(\nu_{X}\right)$. Proposition $3.3(\mathrm{~b})$ then implies that $[T, X]=\left[T, X_{\mathrm{b}}\right]$.

Just as important as the optimal domain space $[T, X]$ is the operator $T$ itself. Proposition 5.2 of [8] implies, for every $f \in L^{1}\left(\nu_{X}\right)$, that $I_{\nu_{X}}(f)$ is the function given by (10). In view of Proposition 3.5 we will, henceforth, interchangeably use both notations $[T, X]$ and $L^{1}\left(\nu_{X}\right)$ without further mention that these spaces coincide, and also the fact that the operators $T$ and $I_{\nu_{X}}$ are equal on $[T, X]=L^{1}\left(\nu_{X}\right)$. 
Proposition 3.6. Let $X$ be a r.i. space not isomorphic to $L^{\infty}([0,1])$. Let $I_{\nu_{X}}: L^{1}\left(\nu_{X}\right) \rightarrow X$ be the integration map of $\nu_{X}$, as in (10).

(a) For $f \in L^{1}\left(\nu_{X}\right)$, we have $\left\|I_{\nu_{X}}(f)\right\|_{X} \leq\|f\|_{\nu_{X}}=\left\|I_{\nu_{X}}(|f|)\right\|_{X}$.

(b) $I_{\nu_{X}}$ is injective.

(c) The range $I_{\nu_{X}}\left(L^{1}\left(\nu_{X}\right)\right)$ is a dense, non-closed subspace of $X_{\mathrm{b}}$. In particular, $I_{\nu_{X}}$ is not surjective.

(d) $I_{\nu_{X}}$ is not compact.

Proof. (a) The stated inequality follows from the fact that $I_{\nu_{X}}$ is bounded with norm at most one. Since $X^{*}$ is a lattice and $\nu_{X}(A) \geq 0$, to prove the equality we argue as in Proposition 2.1(d) to conclude that

$$
\|f\|_{\nu_{X}}=\left\|\int|f| d \nu_{X}\right\|_{X}=\left\|I_{\nu_{X}}(|f|)\right\|_{X}
$$

(b) Let $f \in L^{1}\left(\nu_{X}\right)$. Then $|f| \in L^{1}\left(\nu_{X}\right)$, and so $I_{\nu_{X}}(|f|) \in X$. Since $t \mapsto I_{\nu_{X}}(|f|)(t)$ is decreasing it follows, for every $0<t_{0} \leq 1$, that

$$
\int_{t_{0}}^{1}|f(s)| s^{(1 / n)-1} d s=I_{\nu_{X}}(|f|)\left(t_{0}\right)<\infty .
$$

So, $g(s):=s^{(1 / n)-1} f(s)$ is in $L^{1}\left(\left[t_{0}, 1\right]\right)$. Assume $I_{\nu}(f)=0$ in $X$. Then $I_{\nu}(f)(t)=0$ for a.e. $t \in[0,1]$. Fix $t_{0} \in(0,1)$. For almost all points $a, b$ with $t_{0} \leq a<b \leq 1$, we have $\int_{a}^{b} g d \lambda=I_{\nu}(f)(b)-I_{\nu}(f)(a)=0$. It follows that $g=0$ a.e. in $\left[t_{0}, 1\right]$. Then $g$, and also $f$, is zero a.e. on $[0,1]$.

(c) From (10) it follows that if $f \in L^{1}\left(\nu_{X}\right)$, then $I_{\nu_{X}}(f)$ is an absolutely continuous function on every interval $[t, 1]$, for $0<t \leq 1$. Hence, the Cantor function is in $X$ but not in the range of $I_{\nu_{X}}$. The density of $I_{\nu_{X}}\left(L^{1}\left(\nu_{X}\right)\right)$ in $X_{\mathrm{b}}$ follows from Proposition 2.2(b) and [2, III.6.3].

(d) By Proposition 3.1(a), $F_{X}$ is the Bochner density of the measure $\nu_{X}$ with respect to $\lambda$. Hence, for $f \in L^{1}\left(\nu_{X}\right)$ we have $I_{\nu_{X}}(f)=\int f d \nu_{X}=$ $\int f F_{X} d \lambda$, where the last integral is in the sense of Pettis.

We use the fact $\left[19\right.$, Theorem 1] that $I_{\nu_{X}}: L^{1}\left(\nu_{X}\right) \rightarrow X$ is compact if and only if $G((0,1])$ is relatively compact in $X$, with $G:(0,1] \rightarrow X$ given by $G(s):=F(s) /\|F(s)\|_{X}$. Now,

$$
G(s)=\frac{F(s)}{\|F(s)\|_{X}}=\frac{s^{(1 / n)-1} \chi_{[0, s]}}{s^{(1 / n)-1} \varphi_{X}(s)}=\frac{\chi_{[0, s]}}{\varphi_{X}(s)} .
$$

Assume the set $\left\{\chi_{[0, s]} / \varphi_{X}(s): 0<s \leq 1\right\}$ is relatively compact in $X$. Then there is a sequence $s_{n} \rightarrow 0^{+}$and a function $\psi \in X$ such that the sequence $\chi_{\left[0, s_{n}\right]} / \varphi_{X}\left(s_{n}\right)$ converges to $\psi$ in $X$ and also to $\psi$ pointwise a.e. Since $s_{n} \rightarrow 0^{+}$, this implies $\psi=0$ a.e. on $[0,1]$. But this contradicts the fact that the functions $\chi_{[0, s]} / \varphi_{X}(s)$ all have norm one in $X$. So, $I_{\nu_{X}}$ is not compact. 
REMARK 3.7. The extended operator $T=I_{\nu_{X}}$ is never compact on its optimal domain $[T, X]=L^{1}\left(\nu_{X}\right)$. However, $T: X \rightarrow X$ is compact in most cases of interest. This is the case at least for all spaces $X$ with either $0<\underline{\alpha}$ or $\bar{\alpha}<1$, where $\underline{\alpha}$ and $\bar{\alpha}$ are the Boyd indices of $X$ [2, III.5.12]. This follows from a result of Shimogaki [24], and the fact that $T: L^{\infty}([0,1]) \rightarrow L^{\infty}([0,1])$ is compact (Remark 2.3(b)), as is $T: L^{1}([0,1]) \rightarrow L^{1}([0,1])$ by Remark 3.2.

We end this section with a result concerning the integration map in an important class of r.i. spaces.

Proposition 3.8. Let $\Lambda$ be a Lorentz space. Then the extended operator $T:[T, \Lambda] \rightarrow \Lambda$ is not weakly compact.

Proof. Let $\varphi$ be the function defining the space $\Lambda$. Consider the functions $f_{k}(s):=k s^{1-(1 / n)}(\varphi(s))^{-1} \chi_{(0,1 / k]}(s)$ for $k \geq 1$. Direct computation (via Proposition 3.1) shows that they are in $L^{1}\left(\left|\nu_{\Lambda}\right|\right)$, where they have norm one. Hence $f_{k} \in L^{1}\left(\nu_{\Lambda}\right)=[T, \Lambda]$, where they also have norm one (see Proposition 3.6(a)). Suppose $I_{\nu_{\Lambda}}$ is weakly compact. By passing to a subsequence if necessary, $\left(I_{\nu_{\Lambda}} f_{k}\right)$ converges weakly in $\Lambda$ to a function $\psi \in \Lambda$. By (10) the function $I_{\nu_{A}} f_{k}$ has support in the interval $[0,1 / k]$. It follows that for $0<a<b \leq 1$, we have $\left\langle I_{\nu_{A}} f_{k}, \chi_{[a, b]}\right\rangle \rightarrow 0$. Hence, $\int_{a}^{b} \psi=0$ for every $0<a<b \leq 1$ and so $\psi=0$ a.e. on $[0,1]$.

The function $d \varphi$ belongs to $\Lambda^{\prime}$. On the one hand $\left\langle I_{\nu_{\Lambda}} f_{k}, d \varphi\right\rangle \rightarrow 0$. But this contradicts, for $k \geq 1$, the fact that

$$
\left\langle I_{\nu_{\Lambda}} f_{k}, d \varphi\right\rangle=\int_{0}^{1} d \varphi(t) \int_{t}^{1} \frac{k}{\varphi(s)} \chi_{(0,1 / k]}(s) d s=\int_{0}^{1 / k} \frac{k}{\varphi(s)} \int_{0}^{s} d \varphi(t) d s=1 .
$$

4. The optimal domain for Lorentz $\Lambda$-spaces. We begin with the question: when is $[T, X]$ an AL-space? Recall that a Banach lattice is an abstract $L$-space, for short $A L$-space, if the norm is additive over disjoint elements. By a result of Kakutani every AL-space is $L^{1}(\mu)$ for some measure $\mu$ (see [16]). We include a "folklore" result needed later, but for which we know of no reference.

Lemma 4.1. Let $X$ be a r.i. space on $[0,1]$. If the Lorentz space $\Lambda_{X}$ coincides with $X_{\mathrm{b}}$, then actually $\Lambda_{X}=X$.

Proof. Let $f \in X$. Choose an increasing sequence $\left\{f_{n}\right\}$ of non-negative, bounded functions that converge pointwise to $|f|$. Then

$$
\sup \int_{0}^{1} f_{n}^{*}(s) d \varphi_{X}(s)=\sup \left\|f_{n}\right\|_{\Lambda_{X}}=\sup \left\|f_{n}\right\|_{X} \leq\|f\|_{X}<\infty .
$$

Since $\left\{f_{n}^{*}\right\}$ increases pointwise to $f^{*}$, it follows from Fatou's lemma that $\|f\|_{\Lambda_{X}}=\int_{0}^{1} f^{*}(s) d \varphi_{X}(s)<\infty$. Hence, $f \in \Lambda_{X}$. 
Theorem 4.2. Let $X$ be r.i. Then $[T, X]=L^{1}\left(\nu_{X}\right)$ is order isomorphic to an $A L$-space if and only if $X$ is isomorphic to a Lorentz space.

Proof. If $X$ is isomorphic to $L^{\infty}([0,1])$, then $X=\Lambda_{X}$ and, by Proposition $2.1(\mathrm{~d}), L^{1}\left(\nu_{X}\right)=L^{1}\left(s^{(1 / n)-1} d s\right)$.

Let $X$ be a Lorentz space $\Lambda=\Lambda_{\varphi}$. Fix $f \in L^{1}\left(\left|\nu_{\Lambda}\right|\right)$. By Proposition 3.6(a), (10) and (11),

$$
\begin{aligned}
\|f\|_{L^{1}\left(\nu_{\Lambda}\right)} & =\left\|\int_{(\cdot)}^{1}|f(s)| s^{(1 / n)-1} d s\right\|_{\Lambda}=\iint_{0}^{1}|f(s)| s^{(1 / n)-1} d s d \varphi(t) \\
& =\int_{0}^{1}|f(s)| s^{(1 / n)-1} \varphi(s) d s=\|f\|_{L^{1}\left(\left|\nu_{\Lambda}\right|\right)} .
\end{aligned}
$$

Since $L^{1}\left(\left|\nu_{\Lambda}\right|\right)$ is dense in $L^{1}\left(\nu_{\Lambda}\right)$, we deduce that $L^{1}\left(\nu_{\Lambda}\right)$ and $L^{1}\left(\left|\nu_{\Lambda}\right|\right)$ coincide, and hence $L^{1}\left(\nu_{\Lambda}\right)$ is an AL-space.

Assume $X$ has the property that $L^{1}\left(\nu_{X}\right)$ is order isomorphic to an ALspace. This occurs iff $L^{1}\left(\nu_{X}\right)$ is isomorphic to $L^{1}\left(\left|\nu_{X}\right|\right)$ via the natural inclusion $L^{1}\left(\left|\nu_{X}\right|\right) \hookrightarrow L^{1}\left(\nu_{X}\right)\left[6\right.$, Proposition 2]. Denote $\Lambda_{X}$ by $\Lambda$. Then $\left|\nu_{X}\right|=\left|\nu_{\Lambda}\right|$, as both have density $s^{(1 / n)-1} \varphi_{X}(s)$ with respect to $\lambda$. Thus $L^{1}\left(\left|\nu_{X}\right|\right)$ coincides with $L^{1}\left(\left|\nu_{\Lambda}\right|\right)$. But this last space is isomorphic to $L^{1}\left(\nu_{\Lambda}\right)$. So $L^{1}\left(\nu_{X}\right)$ and $L^{1}\left(\nu_{\Lambda}\right)$ are isomorphic.

Let $f$ be a non-negative function in $L^{1}\left(\nu_{X}\right) \simeq L^{1}\left(\nu_{\Lambda}\right)$. Since $I_{\nu_{X}}=I_{\nu_{\Lambda}}$, we will denote it simply by $I$. By Proposition 3.6(a) we have

$$
\|I(f)\|_{X}=\|f\|_{L^{1}\left(\nu_{X}\right)} \sim\|f\|_{L^{1}\left(\nu_{\Lambda}\right)}=\|I(f)\|_{\Lambda} .
$$

Let $\mathcal{P}$ be the set of all continuous, piecewise affine functions defined on $[0,1]$. Denote by $\mathcal{P}_{1}$ those functions in $\mathcal{P}$ vanishing at 1 . Let $u \in \mathcal{P}_{1}$. Then its decreasing rearrangement $u^{*}$ is also in $\mathcal{P}_{1}$. To see this, first note that if $u$ $\in \mathcal{P}_{1}$, then $|u| \in \mathcal{P}_{1}$. Observe that the distribution function of $u$ is piecewise affine, has no sets of constancy and vanishes at 1 (but is not necessarily continuous). It follows that $u^{*}$ is piecewise affine, continuous and vanishes at 1 , that is, $u^{*} \in \mathcal{P}_{1}$. It follows that $u^{*}$ is absolutely continuous and vanishes at 1 , that is, $u^{*} \in \operatorname{AC}_{1}([0,1])$. By Proposition 2.2(b), there is $f \in L^{1}\left(\nu_{X}\right)$ such that $u^{*}=I(f)$. By injectivity of the integration operator, $f$ is unique. The proof of Proposition 2.2(b) shows that $f(s)=-\left(u^{*}\right)^{\prime}(s) s^{1-(1 / n)}$. Since $u^{*}$ is decreasing, it follows that $f \geq 0$. Both $X$ and $\Lambda$ are r.i. and so, from (15), we have

$$
\|u\|_{X}=\left\|u^{*}\right\|_{X}=\|I(f)\|_{X} \sim\|I(f)\|_{\Lambda}=\left\|u^{*}\right\|_{\Lambda}=\|u\|_{\Lambda} .
$$

Hence, the norms of $X$ and $\Lambda$ are equivalent on $\mathcal{P}_{1}$.

The space $\mathcal{P}$ is dense in $C([0,1])$ in the $X$-norm, since it is so in the uniform norm. Since we are in the situation where $\varphi_{X}\left(0^{+}\right)=0$, the space $C([0,1])$ is dense, for the norm of $X$, in the space $X_{\mathrm{b}}$ [2, III.6.3]. So, $\mathcal{P}$ is 
dense in $X_{\mathrm{b}}$. Given a function in $\mathcal{P}$ we can modify it near 1 to obtain a function still in $\mathcal{P}$ and vanishing at 1 , and so belonging to $\mathcal{P}_{1}$, which is close in the norm of $X\left(X_{\mathrm{b}}\right.$ has a.c. norm as $\varphi_{X}\left(0^{+}\right)=0$ [2, II.5.5]) to the original function [2, I.3.2]. So $\mathcal{P}_{1}$ is dense in $X_{\mathrm{b}}$.

It follows from (16) that the norms of $X$ and $\Lambda=\Lambda_{X}$ are equivalent on $\mathcal{P}_{1}$, which is dense in $X_{\mathrm{b}}$. Hence, the inclusion $\Lambda_{X} \hookrightarrow X_{\mathrm{b}}$ is an isomorphism. Then Lemma 4.1 implies that $X$ is isomorphic to $\Lambda_{X}$.

The following fact was established in the previous proof.

Corollary 4.3. Let $X$ be a Lorentz space. Then $[T, X]=L^{1}\left(\nu_{X}\right)$ is isomorphic to $L^{1}\left(\left|\nu_{X}\right|\right)$. In particular, $L^{1}\left(\nu_{L^{1}}\right)=L^{1}\left(\left|\nu_{L^{1}}\right|\right)=L^{1}\left(s^{1 / n} d s\right)$.

Remark 4.4. Given a r.i. space $X$ with $\varphi_{X}\left(0^{+}\right)=0$, (11) implies for every r.i. space $Y$ with $\varphi_{Y}=\varphi_{X}$ (i.e. $\left.\Lambda_{X} \subset Y \subset M_{X}\right)$ that $\left|\nu_{Y}\right|=\left|\nu_{X}\right|$, and so $L^{1}\left(\left|\nu_{Y}\right|\right)$ coincides with $L^{1}\left(\left|\nu_{X}\right|\right)$. In particular,

$$
L^{1}\left(\nu_{\Lambda_{X}}\right)=L^{1}\left(\left|\nu_{\Lambda_{X}}\right|\right)=L^{1}\left(\left|\nu_{X}\right|\right)=L^{1}\left(\left|\nu_{M_{X}}\right|\right) .
$$

For a vector measure $\nu: \mathcal{B} \rightarrow X$ with values in a r.i. space $X$ on $[0,1]$, it may happen that $L^{1}(\nu) \subset L^{1}([0,1])$, yet $L^{1}(\nu)$ fails to be r.i. [8, Example 5.15]. The space $L^{1}(\nu)$ may also fail to be r.i. simply because it contains $L^{1}([0,1])$ as a proper subspace (Corollary 4.3). Nevertheless, there is always a largest r.i. space inside $L^{1}(\nu)$. If $L^{1}(\nu) \subset L^{1}([0,1])$, this is immediate from [3, Proposition 2.2.6]. Even if $L^{1}(\nu)$ is not inside $L^{1}([0,1])$, a similar construction to that in the proof of Proposition 2.2.6 in [3] still applies to show there is a largest r.i. space inside $L^{1}(\nu)$. Similar comments apply to $L^{1}(|\nu|)$. Summarizing gives

Proposition 4.5. Let $X$ be a r.i. space on $[0,1]$ and $\nu: \mathcal{B} \rightarrow X$ be a vector measure. Then there exist largest r.i. spaces continuously embedded in both $L^{1}\left(\nu_{X}\right)$ and $L^{1}\left(\left|\nu_{X}\right|\right)$.

By Proposition 3.1, the weight function for $L^{1}\left(\left|\nu_{X}\right|\right)$ is $d\left|\nu_{X}\right| / d \lambda:=$ $s^{(1 / n)-1} \varphi_{X}(s)$. The properties of this function and other related ones provide information on various r.i. spaces inside $L^{1}\left(\left|\nu_{X}\right|\right)$. Recall that $n^{\prime}=n /(n-1)$ is the conjugate exponent of $n$.

Proposition 4.6. Let $X$ be a r.i. space. The following properties hold.

(a) $X \subset L^{1}\left(\left|\nu_{X}\right|\right)$ iff $d\left|\nu_{X}\right| / d \lambda \in X^{\prime}$.

(b) $M_{X} \subset L^{1}\left(\left|\nu_{X}\right|\right)$ iff $d\left|\nu_{X}\right| / d \lambda \in \Lambda_{X^{\prime}}$.

(c) $L^{1}([0,1]) \subset L^{1}\left(\left|\nu_{X}\right|\right)$ iff $L_{n^{\prime}, 1}([0,1]) \subset \Lambda_{X}$ iff $d\left|\nu_{X}\right| / d \lambda$ is bounded.

(d) $L^{1}\left(\left|\nu_{X}\right|\right) \subset L^{1}([0,1])$ iff $\Lambda_{X} \subset L_{n^{\prime}, 1}([0,1])$.

Proof. (a) $X \subset L^{1}\left(\left|\nu_{X}\right|\right)$ is equivalent to

$$
\int_{0}^{1}|f(s)| \frac{d\left|\nu_{X}\right|}{d \lambda}(s) d s<\infty \quad \text { for every } f \in X .
$$


This is precisely the condition $d\left|\nu_{X}\right| / d \lambda \in X^{\prime}$.

(b) Since $L^{1}\left(\left|\nu_{X}\right|\right)=L^{1}\left(\left|\nu_{M_{X}}\right|\right)$, by (a) we have $M_{X} \subset L^{1}\left(\left|\nu_{X}\right|\right)$ if and only if $d\left|\nu_{X}\right| / d \lambda \in\left(M_{X}\right)^{\prime}=\Lambda_{X^{\prime}}$.

(c) Since both spaces are Lorentz $\Lambda$-spaces, observe that $Y:=L_{n^{\prime}, 1}([0,1])$ $\subset \Lambda_{X}$ iff there exists $C>0$ such that $\varphi_{X}(s) \leq C \varphi_{Y}(s)=C s^{1 / n^{\prime}}$ on $[0,1]$. This is equivalent to

$$
\frac{d\left|\nu_{X}\right|}{d \lambda}(s)=s^{(1 / n)-1} \varphi_{X}(s) \leq C .
$$

This last condition implies

$$
L^{1}([0,1]) \subset L^{1}\left(\frac{d\left|\nu_{X}\right|}{d \lambda}(s) d s\right)=L^{1}\left(\left|\nu_{X}\right|\right) .
$$

Conversely, if $L^{1}([0,1]) \subset L^{1}\left(\left|\nu_{X}\right|\right)$ then, for a constant $C>0$, we have

$$
\int_{A} \frac{d\left|\nu_{X}\right|}{d \lambda}(s) d s \leq C \int_{A} d s, \quad \text { which implies } \frac{d\left|\nu_{X}\right|}{d \lambda}(s) \leq C .
$$

This is equivalent to $\varphi_{X}(s) \leq C s^{1 / n^{\prime}}$ on $[0,1]$.

(d) Use the same proof as in (c) with inequalities reversed.

For situation (c) above, observe that the largest r.i. space inside $L^{1}\left(\left|\nu_{X}\right|\right)$, and hence also inside $L^{1}\left(\nu_{X}\right)$, is $L^{1}([0,1])$.

Consider now the function $\Theta(t):=\int_{0}^{t} s^{(1 / n)-1} \varphi_{X}(s) d s=\left|\nu_{X}\right|([0, t])$. It is increasing and vanishes at zero. Moreover, $\Theta$ is concave iff $d\left|\nu_{X}\right| / d \lambda$ is decreasing, which is equivalent to the condition

$$
\varphi_{X}^{\prime}(s) \leq \frac{1}{n^{\prime}} \frac{\varphi_{X}(s)}{s}, \quad s \in(0,1] .
$$

Writing (17) as $\varphi_{X}^{\prime}(s) / \varphi_{X}(s) \leq 1 /\left(n^{\prime} s\right)$ and integrating over any subinterval $\left[t_{1}, t_{2}\right] \subset(0,1]$ we see that $(17)$ is equivalent to the function $\varphi_{X}(t) / t^{1 / n^{\prime}}$ being decreasing. Let $0<\alpha \leq 1$. We say that an increasing function $\varphi \geq 0$ is $\alpha$ quasiconcave if $\varphi(t) / t^{\alpha}$ is decreasing. In this case, $s^{\alpha} \leq C \varphi(s)$. Hence, if (17) holds, then $\Lambda_{X} \subset L_{n^{\prime}, 1}([0,1])$, which is the condition of Proposition 4.6(d).

Proposition 4.7. Let $X$ be r.i. with $\varphi_{X}$ being $1 / n^{\prime}$-quasiconcave. Then the Lorentz space $\Lambda_{\Theta}$ is the largest r.i. space inside $L^{1}\left(\left|\nu_{X}\right|\right)$.

Proof. It suffices to show that $\Lambda_{\Theta}$ is in $L^{1}\left(\left|\nu_{X}\right|\right)$ and, if $f \in L^{1}\left(\left|\nu_{X}\right|\right)$ satisfies $f^{*} \in L^{1}\left(\left|\nu_{X}\right|\right)$, then $f \in \Lambda_{\Theta}$.

The hypothesis on $\varphi_{X}$ ensures $\Theta$ is concave. Then $\Theta^{\prime}(s)=s^{(1 / n)-1} \varphi_{X}(s)$ is decreasing and so, by [2, II.2.2],

$$
\int_{0}^{1}|f(s)| s^{(1 / n)-1} \varphi_{X}(s) d s \leq \int_{0}^{1} f^{*}(s) s^{(1 / n)-1} \varphi_{X}(s) d s=\int_{0}^{1} f^{*}(s) d \Theta(s) .
$$


Hence, if $f \in \Lambda_{\Theta}$ then $f \in L^{1}\left(\left|\nu_{X}\right|\right)$. Moreover, if $f \in L^{1}\left(\left|\nu_{X}\right|\right)$ satisfies $f^{*} \in L^{1}\left(\left|\nu_{X}\right|\right)$, then $\left\|f^{*}\right\|_{L^{1}\left(\left|\nu_{X}\right|\right)}=\|f\|_{\Lambda_{\Theta}}$, so $f \in \Lambda_{\Theta}$.

REMARK 4.8. In order to identify the space $\Lambda_{\Theta}$, it is useful to observe that $\Theta$ is equivalent to the function $\Gamma(t):=t^{1 / n} \varphi_{X}(t)$. To see this, recall $\varphi_{X}$ is increasing and concave, hence quasiconcave. Then

$$
\begin{aligned}
n t^{1 / n} \varphi_{X}(t) & =\varphi_{X}(t) \int_{0}^{t} s^{(1 / n)-1} d s \geq \int_{0}^{t} s^{(1 / n)-1} \varphi_{X}(s) d s \\
& \geq \int_{t / 2}^{t} s^{(1 / n)-1} \varphi_{X}(s) d s \geq \frac{t^{1 / n}}{4} \varphi_{X}(t) .
\end{aligned}
$$

Example 4.9. For $X=L^{\infty}([0,1])$, Proposition $2.1(\mathrm{~d})$ implies that $L_{n, 1}([0,1])$ is the largest r.i. space inside $L^{1}\left(\nu_{X}\right)=L^{1}\left(\left|\nu_{X}\right|\right)$.

(a) Consider the spaces $X=L^{p}([0,1])$ for $n^{\prime} \leq p<\infty$. Then $\Theta(t)$ is a multiple of $t^{(1 / n)+(1 / p)}$, which, for the given range of $p$, is concave. Hence, by Proposition 4.7, the largest r.i. space inside $L^{1}\left(\left|\nu_{X}\right|\right)$ is $L_{p_{0}, 1}$, where $p_{0}:=n p /(n+p)$. Actually, in view of Remark 4.4 , the same conclusion holds for $X=L_{p, q}$ with $n^{\prime} \leq p<\infty$ and $1 \leq q \leq \infty$.

(b) For the Lorentz spaces $X=L_{p, 1}$ with $n^{\prime} \leq p<\infty$, the result in (a) also gives the largest r.i. space inside $L^{1}\left(\nu_{X}\right)$ since, by Corollary 4.3, $L^{1}\left(\nu_{X}\right)=L^{1}\left(\left|\nu_{X}\right|\right)$.

(c) Let $X=L^{p}([0,1])$ with $1 \leq p \leq n^{\prime}$. It turns out that

$$
\frac{d\left|\nu_{X}\right|}{d \lambda}(t)=t^{(1 / n)+(1 / p)-1}
$$

is bounded. By Proposition 4.6(c) the largest r.i. space inside $L^{1}\left(\left|\nu_{X}\right|\right)$, and hence also inside $L^{1}\left(\nu_{X}\right)$, is $L^{1}([0,1])$. The same argument holds for $X=L_{p, q}$ with $1 \leq p \leq n^{\prime}$ and $1 \leq q \leq \infty(q \neq \infty$ if $p=1)$.

EXAmple 4.10. Let $X=L \log L$. Since $L_{n^{\prime}, 1}([0,1]) \subset \Lambda_{X}=L \log L$ we see, by Proposition 4.6(c), that the largest r.i. space inside $L^{1}\left(\left|\nu_{X}\right|\right)$ is $L^{1}([0,1])$.

Remark 4.11. In Proposition 4.7 it is enough if $\varphi_{X}$ is essentially $1 / n^{\prime}$ quasiconcave, that is, if condition (17) is satisfied on $[0, \delta]$ for some $0<\delta<1$. For, in this case, there is a concave function $\widetilde{\Theta}$ which coincides with $\Theta$ on $[0, \delta]$ and $\widetilde{\Theta}$ and $\widetilde{\Theta}^{\prime}$ are equivalent to $\Theta$ and $\Theta^{\prime}$, respectively. Hence, the spaces $\Lambda_{\Theta}$ and $\Lambda_{\widetilde{\Theta}}$ are isomorphic.

ExAmple 4.12. For $1 \leq p<\infty$, consider the Zygmund spaces $X=$ $\operatorname{Exp} L^{p}$ of functions $f$ of $p$ th exponential integrability, that is, with $\int_{0}^{1} \exp (\alpha|f|)^{p}<\infty$ for some $\alpha=\alpha(f)>0$ (i.e. the Orlicz space given by the function $\left.\Phi(t)=\exp \left(t^{p}\right)-1\right)$ [2, IV.6.11]. Then $\varphi_{X}(t)=\log ^{-1 / p}(1+1 / t)$ and so $(17)$ is satisfied on $[0, \delta]$ for a certain $\delta$ depending on $n$ and $p$. 
Hence, by Remark 4.11, the largest r.i. space inside $L^{1}\left(\left|\nu_{X}\right|\right)$ is $\Lambda_{\Gamma}$ for $\Gamma(t)=t^{1 / n} \log ^{-1 / p}(1+1 / t)$; see Remark 4.8 .

5. Optimal domain for Marcinkiewicz spaces. We now consider the problem of finding large r.i. spaces inside $L^{1}\left(\nu_{X}\right)=[T, X]$. Proposition 4.6(c) implies that $L^{1}([0,1])$ is the largest r.i. space inside $L^{1}\left(\nu_{X}\right)$ whenever the function $d\left|\nu_{X}\right| / d \lambda$ is bounded.

We need to invoke the dual operator $W$ of the integration map $I_{\nu_{X}}$, namely $W g(s):=s^{(1 / n)-1} \int_{0}^{s} g(t) d t$, for $g \in X^{\prime}$. If $H$ denotes the HardyLittlewood operator, defined by $H h(s):=s^{-1} \int_{0}^{s} h(t) d t$, then $W g(s)=$ $s^{1 / n} H g(s)$.

Proposition 5.1. Let $X$ be a r.i. space. Then $X \subset[T, X]$.

Proof. Direct computation shows that the operator $W$ is continuous from $L^{1}([0,1])$ into $L^{1}([0,1])$ and from $L^{\infty}([0,1])$ into $L^{\infty}([0,1])$. Hence, by the interpolation theorem, it maps $X^{\prime}$ into $X^{\prime}$. So, if $g \in X^{\prime}$ is non-negative and decreasing and $f \in X$, then $\int_{0}^{1}|f(s)| W g(s) d s<\infty$. Accordingly, (12) is satisfied and so $f \in L^{1}\left(\nu_{X}\right)$.

REmark 5.2. The inclusion $X \subset[T, X]$ is always proper whenever $T$ : $X \rightarrow X$ is compact; see Remark 3.7.

Now we can consider a "missing" part in Proposition 4.6, equivalent to the condition $d\left|\nu_{X}\right| / d \lambda \in M_{X^{\prime}}$.

Corollary 5.3. Let $X$ be a r.i. space. Then $\Lambda_{X} \subset L^{1}\left(\left|\nu_{X}\right|\right)$.

Proof. For $X=\Lambda_{X}$, Proposition 5.1 gives $\Lambda_{X} \subset L^{1}\left(\nu_{\Lambda_{X}}\right)$ and by Remark 4.4 we have $L^{1}\left(\nu_{\Lambda_{X}}\right)=L^{1}\left(\left|\nu_{X}\right|\right)$.

REMARK 5.4. The largest r.i. space inside $\left[T, L^{\infty}([0,1])\right]$ is $L_{n, 1}([0,1])$; see Example 4.9. Since $L^{\infty}([0,1]) \subset X$, we have $\left[T, L^{\infty}([0,1])\right] \subset[T, X]$. So, by Proposition 5.1 , we have in fact $X+L_{n, 1}([0,1]) \subset[T, X][13$, p. 9]. This implies that $W: X^{\prime} \rightarrow X^{\prime} \cap L_{n^{\prime}, \infty}$.

In view of Proposition 5.1 one may also ask: when does the inclusion $M_{X} \subset[T, X]$ hold? Proposition 4.6(b) shows that if $d\left|\nu_{X}\right| / d \lambda \in \Lambda_{X^{\prime}}$ then $M_{X} \subset L^{1}\left(\left|\nu_{X}\right|\right) \subset L^{1}\left(\nu_{X}\right)$.

Proposition 5.5. For $X$ a r.i. space, $M_{X} \subset[T, X]$ iff $W: X^{\prime} \rightarrow \Lambda_{X^{\prime}}$.

Proof. If $W: X^{\prime} \rightarrow \Lambda_{X^{\prime}}$ and $f \in M_{X}$, we have $\int_{0}^{1}|f(s)| W g(s) d s<\infty$ for every $g \in X^{\prime}$ non-negative and decreasing. Hence, (12) is satisfied and so $f \in L^{1}\left(\nu_{X}\right)$. Conversely, if $M_{X} \subset L^{1}\left(\nu_{X}\right)$, then (12) yields $\int_{0}^{1}|f(s)| W g(s) d s$ $<\infty$ for each $f \in M_{X}$ and every $0 \leq g \in X^{\prime}$ decreasing, hence for all $g \in X^{\prime}$. This implies $W g \in \Lambda_{X^{\prime}}$. 
We now consider the optimal domain when the kernel operator $T$ takes its values in a Marcinkiewicz space $X=M_{\varphi}$. Recall that the lower dilation exponent $\gamma_{\varphi}$ and the upper dilation exponent $\delta_{\varphi}$ of the function $\varphi$ (see $[13$, p. 54]) are given by

$$
\gamma_{\varphi}:=\lim _{t \rightarrow 0^{+}} \frac{\log \sup _{s}\{\varphi(s t) / \varphi(s)\}}{\log t}, \quad \delta_{\varphi}:=\lim _{t \rightarrow \infty} \frac{\log \sup _{s}\{\varphi(s t) / \varphi(s)\}}{\log t} .
$$

For $\varphi$ increasing and concave we have $0 \leq \gamma_{\varphi} \leq \delta_{\varphi} \leq 1$ [13, p. 54]. Since $\int_{0}^{t} f^{*}(s) d s \geq t f^{*}(t)$, in general the norm of $f$ in $M_{\varphi}$ is greater than or equal to the expression

$$
\sup _{t} \frac{t}{\varphi(t)} f^{*}(t)
$$

However, if $\gamma_{\varphi}>0$, the norm of $f \in M_{\varphi}$ is equivalent to (18) [13, pp. 114-115 and 56-57]. When $\varphi=\varphi_{X}$, for a r.i. space $X$, the dilation exponents are the fundamental or Zippin indices of $X$; see [2, p. 177] and [17, p. 27].

The next lemma (extending [13, (2.40), p. 75]) is needed later.

LEMMA 5.6. Let $\alpha>0$ and let $\varphi$ be an increasing, concave function satisfying $\varphi(0)=0$ and $\gamma_{\varphi}>\alpha$. Then there exists a constant $C>0$, depending only on $\alpha$ and $\varphi$, such that

$$
\int_{0}^{1}\left(s^{\alpha} g(s)\right)^{*} s^{-\alpha} \varphi^{\prime}(s) d s \leq C \int_{0}^{1} g(s) \varphi^{\prime}(s) d s,
$$

for every decreasing function $g$ on $[0,1]$.

Proof. Following the proof of [13, (2.40), p. 75], it is enough to show that the result holds for $g=\chi_{(0, a]}$. In this case

$$
\begin{aligned}
\int_{0}^{1}\left(s^{\alpha} \chi_{(0, a]}(s)\right)^{*} s^{-\alpha} \varphi^{\prime}(s) d s & =\sum_{k=1}^{\infty} \int_{a /(k+1)}^{a / k}\left(\frac{a-s}{s}\right)^{\alpha} \varphi^{\prime}(s) d s \\
& \leq \sum_{k=1}^{\infty} k^{\alpha}\left(\varphi\left(\frac{a}{k}\right)-\varphi\left(\frac{a}{k+1}\right)\right) \\
& =\varphi(a)+\sum_{k=2}^{\infty} \varphi\left(\frac{a}{k}\right)\left(k^{\alpha}-(k-1)^{\alpha}\right) \\
& \leq \varphi(a) C_{1}\left(1+\sum_{k=2}^{\infty} \frac{\varphi(a / k)}{\varphi(a)} k^{\alpha-1}\right)
\end{aligned}
$$

where $C_{1}$ is a constant depending on $\alpha$. Since $\gamma_{\varphi}>\alpha$, for any $0<\varepsilon<$ $\gamma_{\varphi}-\alpha$ there exists $\delta>0$ such that $(\log t)^{-1} \log \sup _{s}\{\varphi(s t) / \varphi(s)\}>\alpha+\varepsilon$ if $0<t<\delta$. So, for every $a \in(0,1]$, we have $t^{\alpha+\varepsilon}>\varphi(a t) / \varphi(a)$, provided $0<t<\delta$. Hence, there is $k_{0} \geq 1$ with 


$$
\sum_{k=2}^{\infty} \frac{\varphi(a / k)}{\varphi(a)} k^{\alpha-1} \leq C_{2}=\sum_{k=2}^{k_{0}} k^{\alpha-1}+\sum_{k>k_{0}} k^{-\varepsilon-1}<\infty .
$$

Accordingly, for $C=C_{1} C_{2}$, which does not depend on $a$, we have

$$
\int_{0}^{1}\left(s^{\alpha} \chi_{(0, a]}(s)\right)^{*} s^{-\alpha} \varphi^{\prime}(s) d s \leq C \varphi(a)=C \int_{0}^{1} \chi_{(0, a]}(s) \varphi^{\prime}(s) d s .
$$

Theorem 5.7. Consider a Marcinkiewicz space $M_{\varphi}$ with $\varphi$ satisfying $1 / n<\gamma_{\varphi} \leq \delta_{\varphi}<1$. Then the largest r.i. space inside $\left[T, M_{\varphi}\right]$ is the Marcinkiewicz space $M_{\Phi}$, where $\Phi(t):=\int_{0}^{t} s^{-1 / n} \varphi^{\prime}(s) d s$. Under these conditions, $\Phi$ is equivalent to $\Psi(t):=t^{-1 / n} \varphi(t)$.

Proof. The function $\Phi$ is increasing and concave. We first prove its equivalence with $\Psi$. One inequality is immediate as

$$
\Psi(t)=t^{-1 / n} \int_{0}^{t} \varphi^{\prime}(s) d s \leq \int_{0}^{t} s^{-1 / n} \varphi^{\prime}(s) d s=\Phi(t) .
$$

For the other inequality, using $\gamma_{\varphi}>1 / n$, a direct computation shows that $\gamma_{\Psi}>0$. Then $\Psi$ is equivalent to the function $t \mapsto \int_{0}^{t}(\Psi(s) / s) d s[13$, p. 57]. This, together with $\varphi^{\prime}(t) \leq \varphi(t) / t$ (see [17, (4.9), p. 26]), implies, for some constant $M>0$, that

$$
\Phi(t)=\int_{0}^{t} s^{-1 / n} \varphi^{\prime}(s) d s \leq \int_{0}^{t} \frac{\Psi(s)}{s} d s \leq M \Psi(t) .
$$

Hence, $\Phi$ and $\Psi$ are equivalent. In particular, $\gamma_{\Phi}=\gamma_{\Psi}>0$.

Let $f \in M_{\Phi}$. Let $g \in \Lambda_{\varphi}=\left(M_{\varphi}\right)^{\prime}$ be non-negative and decreasing. The condition $\delta_{\varphi}<1$ ensures that the Hardy-Littlewood operator $H$ is continuous from $\Lambda_{\varphi}$ into $\Lambda_{\varphi}[13$, p. $138 ;(4.20)$, p. 99; (1.20), p. 54]. Hence, $H g \in \Lambda_{\varphi}$. Moreover, $g$ decreasing implies $H g$ is also decreasing. Since $\Phi^{\prime}(t)=$ $t^{-1 / n} \varphi^{\prime}(t)$ and $\gamma_{\varphi}>1 / n$, by Lemma 5.6 we have

$$
\int_{0}^{1}\left(s^{1 / n} H g(s)\right)^{*} d \Phi(s) \leq C \int_{0}^{1} H g(s) d \varphi(s) .
$$

This last integral is finite because $(H g)^{*}=H g$ and $H g \in \Lambda_{\varphi}$. So $s^{1 / n} H g(s)$ $\in \Lambda_{\Phi}$. Then, via the $\left\langle\Lambda_{\Phi}, M_{\Phi}\right\rangle$ duality, we see that

$$
\int_{0}^{1}|f(s)| s^{(1 / n)-1} \int_{0}^{s} g(t) d t d s=\left\langle f, s^{1 / n} H g(s)\right\rangle \leq\|f\|_{M_{\Phi}}\left\|s^{1 / n} H g(s)\right\|_{\Lambda_{\Phi}}
$$

is finite. According to (12), we have $f \in\left[T, M_{\varphi}\right]$.

Let $f \in\left[T, M_{\varphi}\right]$ satisfy $f^{*} \in\left[T, M_{\varphi}\right]$. Then $T\left(f^{*}\right) \in M_{\varphi}$. By (10), $T\left(f^{*}\right)$ is decreasing and so $\sup _{t}(t / \varphi(t)) \int_{t}^{1} f^{*}(s) s^{(1 / n)-1} d s=: A<\infty$. Thus, for 
every $t \in(0,1]$,

$$
A \geq \frac{t / 2}{\varphi(t / 2)} \int_{t / 2}^{t} f^{*}(s) s^{(1 / n)-1} d s \geq \frac{t / 2}{\varphi(t / 2)} f^{*}(t) t^{(1 / n)-1} \frac{t}{2} \geq \frac{t^{1 / n}}{4 \varphi(t)} f^{*}(t) t .
$$

Hence, $\sup _{t}(t / \Psi(t)) f^{*}(t)<\infty$. Since $\Psi$ and $\Phi$ are equivalent, it follows that $\sup _{t}(t / \Phi(t)) f^{*}(t)<\infty$. We already observed that $\gamma_{\Phi}>0$. So $(18)$, with $\Phi$ in place of $\varphi$, is equivalent to the norm of $M_{\Phi}$, and hence $f \in M_{\Phi}$. Since $M_{\Phi}$ is itself r.i., this implies $M_{\Phi}$ is the largest r.i. space inside $\left[T, M_{\varphi}\right]$.

Corollary 5.8. Let $\varphi$ be as in Theorem 5.7. Then $\left[T, M_{\varphi}\right]$ is not r.i.

Proof. From the proof of Theorem 5.7, $\Phi$ and $t \mapsto \int_{0}^{t}(\Phi(s) / s) d s$ are equivalent. This implies that $f(t):=\Phi(t) / t \in M_{\Phi}$. Set $f_{n}:=\min \{f, n\}$. Then $f_{n}$ increases to $f$, but direct computation shows that $\left\|f-f_{n}\right\|_{M_{\Phi}}$ does not converge to zero. Hence, $M_{\Phi}$ does not have a.c. norm. But the space $\left[T, M_{\varphi}\right]$ does have a.c. norm (Proposition 3.3(a)). Hence, $M_{\Phi} \subsetneq\left[T, M_{\varphi}\right]$, showing that $\left[T, M_{\varphi}\right]$ is not r.i.

ExAMPLE 5.9. The space $L_{p, \infty}$, for $1<p<\infty$, is a Marcinkiewicz space with $\varphi(t)=t^{1 / p^{\prime}}$, where $p^{\prime}$ is the conjugate exponent of $p$. The condition $\gamma_{\varphi}>1 / n$ is precisely $p>n^{\prime}$. If we set $p_{0}:=n p /(n+p)$, the previous condition is $p_{0}>1$. The condition $\delta_{\varphi}<1$ is $p<\infty$, which is $p_{0}<n$. Under these conditions, the function $\Phi$ of Theorem 5.7 is equivalent to $t^{1 / p_{0}^{\prime}}$. So, the largest r.i. space inside $\left[T, L_{p, \infty}\right]$ is $L_{p_{0}, \infty}$.

REMARK 5.10. For $X=L_{p, \infty}$ with $1<p \leq n^{\prime}$, the function $\Phi$ in Theorem 5.7 is everywhere infinite. In this case it can be shown that

$$
\sup _{t} t^{1 / p} T\left(f^{*}\right)(t)<\infty \Leftrightarrow \sup _{t} t^{1 / p_{0}} f^{*}(t)<\infty
$$

For $p=n^{\prime}$, this yields $f^{*} \in\left[T, L_{n^{\prime}, \infty}\right]$ iff $f^{*} \in L_{1, \infty}$, that is, weak- $L^{1}$ is the largest r.i. quasi-Banach space inside $\left[T, L_{n^{\prime}, \infty}\right]$.

Under certain circumstances, we can use the previous results to give a description of the largest r.i. space inside $[T, X]$ when $X$ is not a Marcinkiewicz space, by identifying its fundamental function. Our last result is of this kind.

THEOREM 5.11. Let $X$ be a r.i. space such that $\varphi_{X}$ is $1 / n^{\prime}$-quasiconcave and $0<\gamma_{\varphi_{X}} \leq \delta_{\varphi_{X}}<1 / n^{\prime}$. Then the largest r.i. space inside $[T, X]$ has fundamental function equivalent to $\Gamma(t)=t^{1 / n} \varphi_{X}(t)$.

Proof. We first identify the largest r.i. spaces inside $\left[T, \Lambda_{X}\right]$ and $\left[T, M_{X}\right]$. We are in the situation of Proposition 4.7 and so $\Lambda_{\Theta}$ is the largest r.i. space inside $\left[T, \Lambda_{X}\right]$, with $\Theta$ equivalent to $\Gamma$; see Remark 4.8.

The Marcinkiewicz space associated to $X$ is $M_{X}=M_{\varphi}$ with $\varphi(t):=$ $t / \varphi_{X}(t)$. The conditions $0<\gamma_{\varphi_{X}} \leq \delta_{\varphi_{X}}<1 / n^{\prime}$ imply that $1 / n<\gamma_{\varphi} \leq$ $\delta_{\varphi}<1$ [2, p. 178]. We are in the situation of Theorem 5.7 and so the 
Marcinkiewicz space $M_{\Phi}$ is the largest r.i. space inside $\left[T, M_{X}\right]$, with $\Phi$ equivalent to $\Psi(t)=t /\left(t^{1 / n} \varphi_{X}(t)\right)$.

Since $\Psi(t) \Gamma(t)=t$, we deduce that $\Theta$ and $t / \Phi(t)$ are equivalent. This implies that $M_{\Phi}$ is isomorphic to the Marcinkiewicz space associated to the function $t / \Theta(t)$. Hence, $\Lambda_{\Theta}$ and $M_{\Phi}$ are (isomorphic to) the smallest and the largest r.i. spaces with fundamental function $\Theta$, respectively.

Let $Y \subset[T, X]$ be the largest r.i. space inside $[T, X]$; see Proposition 4.5. Since $\left[T, \Lambda_{X}\right] \subseteq[T, X] \subseteq\left[T, M_{X}\right]$, the above discussion shows that $\Lambda_{\Theta} \subset Y \subset M_{\Phi}$. So $\varphi_{Y}$ is equivalent to $\Theta$, hence to $\Gamma$.

REMARK 5.12. It is useful to observe that if $\varphi$ is $\alpha$-quasiconcave, then $\delta_{\varphi} \leq \alpha$ (by direct calculation). Hence, in the above theorem it suffices to require that $\gamma_{\varphi_{X}}>0$ and $\varphi_{X}$ is $\alpha$-quasiconcave for some $\alpha<1 / n^{\prime}$.

ExAMPLE 5.13. In view of Theorem 5.11 (and Examples 4.9 and 5.9), if $X=L_{p, q}$ for $n^{\prime}<p<\infty$ and $1 \leq q \leq \infty$, then the largest r.i. space inside $[T, X]$ has fundamental function equivalent to $t^{1 / p_{0}}$. Observe that $p=n p_{0} /\left(n-p_{0}\right)$ is the Sobolev exponent corresponding to $p_{0}$.

\section{References}

[1] R. G. Bartle, N. Dunford and J. Schwartz, Weak compactness and vector measures, Canad. J. Math. 7 (1955), 289-305.

[2] C. Bennett and R. Sharpley, Interpolation of Operators, Academic Press, Boston, 1988.

[3] Yu. A. Brudnyı and N. Ya. Krugljak, Interpolation Functors and Interpolation Spaces, North-Holland, Amsterdam, 1991.

[4] A. Cianchi, A sharp embedding theorem for Orlicz-Sobolev spaces, Indiana Univ. Math. J. 45 (1996), 39-65.

[5] G. P. Curbera, Operators into $L^{1}$ of a vector measure and application to Banach lattices, Math. Ann. 293 (1992), 317-330.

[6] -, When $L^{1}$ of a vector measure is an AL-space, Pacific J. Math. 162 (1994), 287-303.

[7] -, A note on function spaces generated by Rademacher series, Proc. Edinburgh Math. Soc. 40 (1997), 119-126.

[8] G. P. Curbera and W. J. Ricker, Optimal domains for kernel operators via interpolation, Math. Nachr. 244 (2002), 47-63.

[9] J. Diestel and J. J. Uhl, Jr., Vector Measures, Amer. Math. Soc., Providence, RI, 1977.

[10] D. Edmunds, R. Kerman and L. Pick, Optimal Sobolev imbeddings involving rearrangement-invariant quasinorms, J. Funct. Anal. 170 (2000), 307-355.

[11] J. A. Hempel, G. R. Morris and N. S. Trudinger, On the sharpness of a limiting case of the Sobolev imbedding theorem, Bull. Austral. Math. Soc. 3 (1970), 369-373.

[12] R. Huff, Remarks on Pettis integrability, Proc. Amer. Math. Soc. 96 (1986), 402-404.

[13] S. G. Kreı̆n, Yu. I. Petunin and E. M. Semënov, Interpolation of Linear Operators, Amer. Math. Soc., Providence, RI, 1982. 
[14] D. R. Lewis, Integration with respect to vector measures, Pacific J. Math. 33 (1970), $157-165$.

[15] - On integration and summability in vector spaces, Illinois J. Math. 16 (1972), 294-307.

[16] J. Lindenstrauss and L. Tzafriri, Classical Banach Spaces, Vol. II, Springer, Berlin, 1979.

[17] L. Maligranda, Indices and interpolation, Dissertationes Math. 234 (1985).

[18] S. Okada, The dual space of $\mathcal{L}^{1}(\mu)$ for a vector measure $\mu$, J. Math. Anal. Appl. 177 (1993), 583-599.

[19] S. Okada, W. J. Ricker and L. Rodríguez-Piazza, Compactness of the integration operator associated with a vector measure, Studia Math. 150 (2002), 133-149.

[20] L. Pick, Optimal Sobolev Embeddings, Rudolph-Lipschitz-Vorlesungsreihe Nr. 43, SFB 256: Nichtlineare Partielle Differentialgleichungen, Univ. of Bonn, 2002.

[21] W. J. Ricker, Separability of the $L^{1}$-space of a vector measure, Glasgow Math. J. 34 (1992),1-9.

[22] - Compactness properties of extended Volterra operators in $L^{p}([0,1])$ for $1 \leq p$ $\leq \infty$, Arch. Math. (Basel) 66 (1996), 132-140.

[23] V. A. Rodin and E. M. Semënov, Rademacher series in symmetric spaces, Anal. Math. 1 (1975), 207-222.

[24] T. Shimogaki, On the complete continuity of operators in an interpolation theorem, J. Fac. Sci. Hokkaido Univ. Ser. I 20 (1968), 109-114.

[25] N. S. Trudinger, On imbeddings into Orlicz spaces and some applications, J. Math. Mech. 17 (1967), 473-484.

Facultad de Matemáticas

Universidad de Sevilla

Aptdo. 1160, Sevilla 41080, Spain

E-mail: curbera@us.es
Math.-Geogr. Fakultät Katholische Universität Eichstätt-Ingolstadt D-85072 Eichstätt, Germany E-mail: werner.ricker@ku-eichstaett.de

Received June 27, 2002

Revised version April 4, 2003 